\title{
Up-to-Date Finite Element Based Simulation of Permanent Magnet
}

\author{
G. Kovács \\ Széchenyi István University, Department of Automation \\ Egyetem tér 1, H-9026 Győr, Hungary \\ e-mail: kovacsg@maxwell.sze.hu
}

Abstract: The paper presents a two dimensional finite element based solver for static magnetic field problems. The application has been written in $\mathrm{C}$ programming language. Magnetic vector potential of some reference models have been calculated by the help of two environments. The first one is the MATLAB environment and the second one is the $\mathrm{C}$ programming language based finite element code. The simulation results of the two environments were compared to each other focusing on the magnetic vector potential and the simulation time. Differences of the simulation results are showed in this paper, as well.

Keywords: Finite Element Method, C programming language, MATLAB, permanent magnet

\section{Introduction}

The computer-aided design is one of the important parts of the electric engine development. Electric engines have been improved at the Széchenyi István University, as well and one of the parts of this development is to design and optimization a brushless DC (direct current) motor family, which are will be applied with bicycles and smaller motors. There are also two projects where these motors will be applied. One of them is the development of hybrid E-VAN, which is an adapted Ford-truck. The second one is an electric car which is an individual development by the students and teachers of the university. The main aspect of this PMS motor (Permanent Magnet Synchronous motor) development is to reduce the weight and the size of the engine but the torque and losses of the motor should not decrease. There are different ways to design the PMS motors. This paper shows a $\mathrm{C}$ programming language based finite element simulation of the permanent magnets which are used in permanent magnet synchronous motors.

\section{Structure of the development environment}

The new finite element package consists of two main parts which can be seen in Fig. 1 . The first part is the GMSH software [1] which is a two and three-dimensional finite element mesh generator with built-in pre- and post-processing facilities. The second part of the finite element development environment is the $\mathrm{C}$ language based package under 
Linux operating system, where partial differential equations and matrix operations can be solved. The finite element based solver has been written by the help of this package.

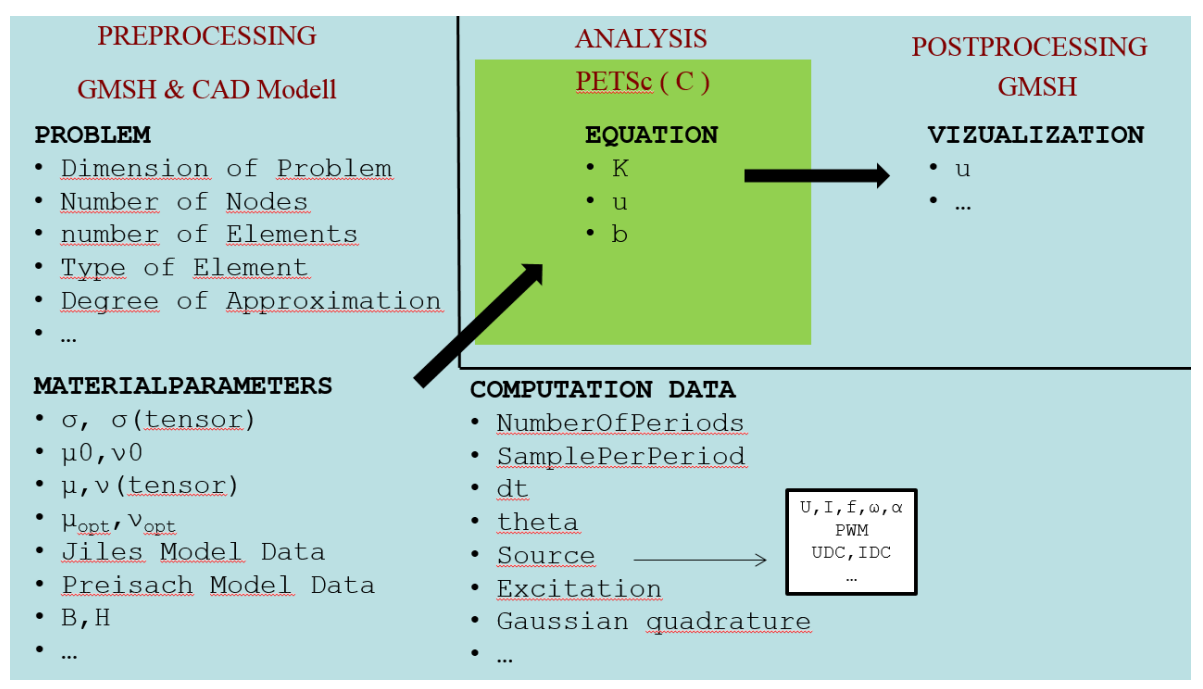

Figure 1. Structure of the development environment

Many data have to be used in the FEM (Finite Element Method) structure. For example number of nodes, number of elements, material parameters, etc., which are given from the geometry and the mesh in the pre-procession step? These parameters and data are used in the Analysis step to solve the equations of partial differential equations. And finally the data of the simulation results are shown in the post-procession step.

Fig. 2. shows the graphical user interface of the finite element based package which consists of two parts.

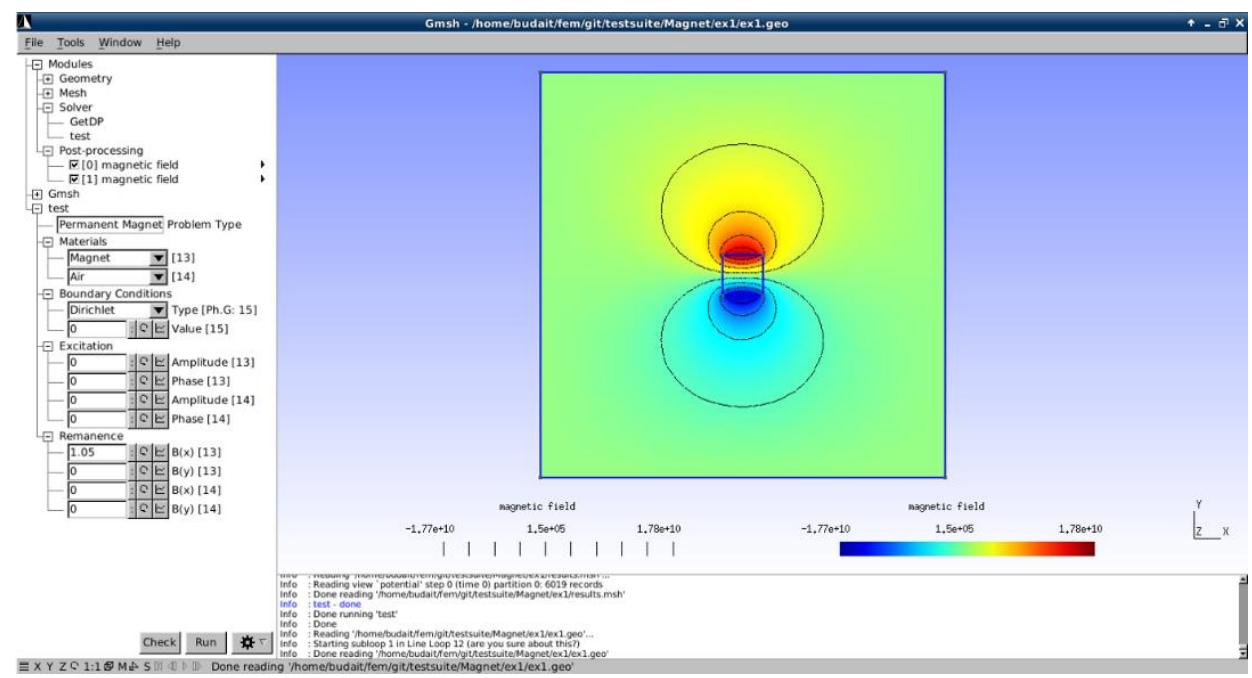

Figure 2. Graphical User Interface 
On the left side the mesh, material parameters and boundary conditions can be set. And on the right side the model and the simulation results can be seen which a permanent magnet is.

\section{Governing Equations}

The basic model consists of a permanent magnet, a ferrite core and an excited single coil which can be seen in Fig. 3.

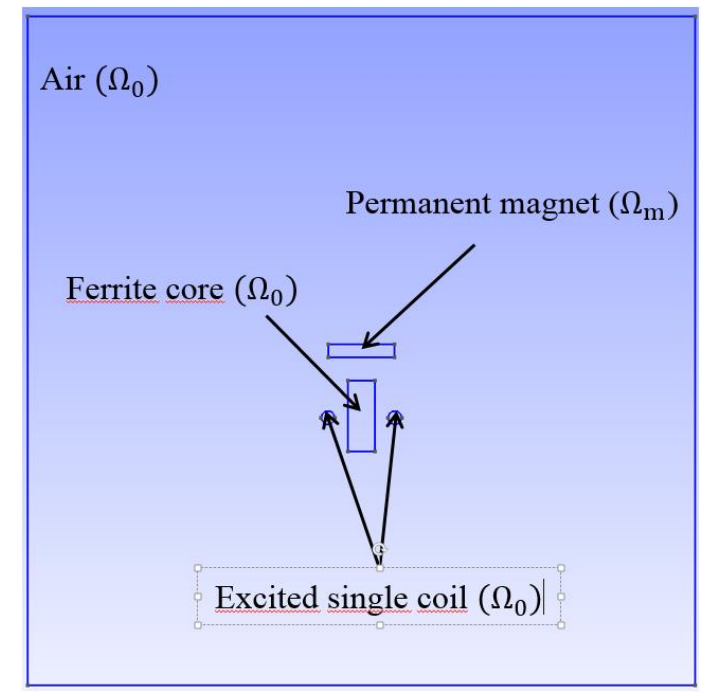

Figure 3. Scheme of the basic model

From the basic model five simple different arrangements had been created. The different simulated arrangements are as follows:

- $\quad$ One turned excited coil;

- $\quad$ One turned excited coil with ferrite core;

- Permanent magnet;

- Permanent magnet with ferrite core;

- $\quad$ The basic model which contains the permanent magnet, the ferrite core and the excited coil, as well.

These models have been calculated in the $\mathrm{C}$ programing language based the finite element environment and in MATLAB environment.

The simulated problem has been modelled as a static magnetic field problem, where the following Maxwell's equations can be used [2]-[5]:

$$
\begin{aligned}
\nabla \times \boldsymbol{H} & =\boldsymbol{J}_{0}, \text { in } \Omega_{0} \cup \Omega_{\mathrm{m}}, \\
\nabla \cdot \boldsymbol{B} & =0, \text { in } \Omega_{0} \cup \Omega_{\mathrm{m}} .
\end{aligned}
$$


Here $\boldsymbol{H}$ is the magnetic field intensity, $\boldsymbol{J}_{\mathbf{0}}$ is the source current density, $\boldsymbol{B}$ is the magnetic flux density. The $\boldsymbol{H}$ magnetic field intensity can be expressed as

$$
\boldsymbol{H}=\left\{\begin{array}{c}
v_{0} \boldsymbol{B}, \text { in } \Omega_{0} \\
v_{0} v_{\mathrm{r}} \boldsymbol{B} \text { in } \Omega_{\mathrm{m}}
\end{array} .\right.
$$

Here $v_{0}$ is the reluctivity of vacuum and $v_{\mathrm{r}}$ is the relative reluctivity of magnet. The air region is denoted by $\Omega_{0}$ and the magnetic region is denoted by $\Omega_{\mathrm{m}}$. The $\boldsymbol{A}$ magnetic flux density can be expressed as

$$
\boldsymbol{B}=\nabla \times \boldsymbol{A},
$$

where $\boldsymbol{A}$ is the magnetic vector potential [2]-[5]. This expression satisfies (2), because of the identity $\nabla \cdot \nabla \times \boldsymbol{v}$ for any vector function $\boldsymbol{v}=\boldsymbol{v}(\boldsymbol{r})$.

When the domain contains permanent magnets, their magnetic characteristic are given by [3]

$$
\boldsymbol{B}=\mu \boldsymbol{H}+\boldsymbol{B}_{0},
$$

where $\boldsymbol{B}_{0}$ is the remanent flux density. Substituting (1) and (4) to (5) and using the constitutive relations IS (3) the following partial differential equation can be obtained:

$$
\nabla \times \frac{1}{\mu} \nabla \times \boldsymbol{A}-\nabla \times \frac{1}{\mu} \boldsymbol{B}_{0}=\boldsymbol{J}_{0} .
$$

The divergence of the magnetic vector potential can be selected according to Coulomb's gauge [5,7],

$$
\nabla \cdot \boldsymbol{A}=0
$$

which is satisfied automatically in two dimensional problems [4], [5]. Using some mathematical identity and using some formulations [4], [5], the following weak equation can be obtained

$$
\int_{\Omega} \frac{1}{\mu} \nabla \times \boldsymbol{W} \cdot \nabla \times \boldsymbol{A} \mathrm{d} \Omega-\int_{\Omega} \frac{1}{\mu} \nabla \times \boldsymbol{W} \cdot \boldsymbol{B}_{0} \mathrm{~d} \Omega=\int_{\Omega} \boldsymbol{W} \cdot \boldsymbol{J}_{0} \mathrm{~d} \Omega,
$$

which solution results in the approximation of the magnetic vector potential.

\section{Simulation results of the models}

Five simple different arrangements had been created from the basic model. These models are calculated in the $\mathrm{C}$ programing language based on the finite element environment and in MATLAB environment. The simulation results were compared each other focusing the time and the accurate of the magnetic vector potential. In both environments the unknowns are the same, or closely the same which is 12790 .

\subsection{Comparison of the computation time of the models}

Computation times of two different development environment are compared with each other. The sum up of the computation times of the simulations can be seen in Table 1. 
Table 1. Computation times of models in two different environments

\begin{tabular}{|c|c|c|}
\hline model & MATLAB & $\boldsymbol{C}$ \\
\hline excited coil & $60 \mathrm{sec}$ & $13 \mathrm{sec}$ \\
\hline ferrite core and excited coil & $60 \mathrm{sec}$ & $13 \mathrm{sec}$ \\
\hline magnet & $60 \mathrm{sec}$ & $12 \mathrm{sec}$ \\
\hline magnet and ferrite core & $59 \mathrm{sec}$ & $10 \mathrm{sec}$ \\
\hline magnet, ferrite core and excited coil & $63 \mathrm{sec}$ & $11 \mathrm{sec}$ \\
\hline
\end{tabular}

On the left side the computation times in MATLAB environment are shown, and on the right side the computation times in $\mathrm{C}$ environment are shown in Table 1 . In case of every arrangement the problem was calculated almost five times faster by the help of the $\mathrm{C}$ environment than with MATLAB.

\subsection{Comparison of the magnetic vector potential of the models}

Simulation results were also compared with each other focusing on the accuracy of the magnetic vector potential. Fig. 4. shows the simulation result of the magnetic potential in the case of excited one turned coil model. Fig 4a shows the simulation results in GMSH environment. Fig. 4b shows the simulation results in MATLAB environment.

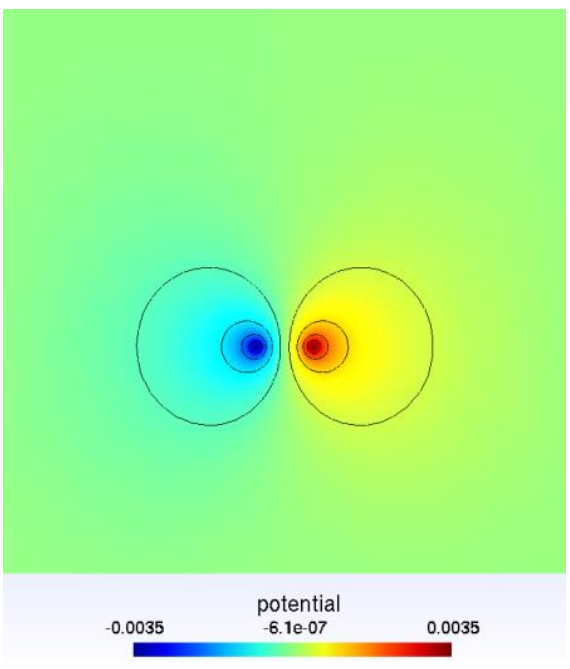

a)

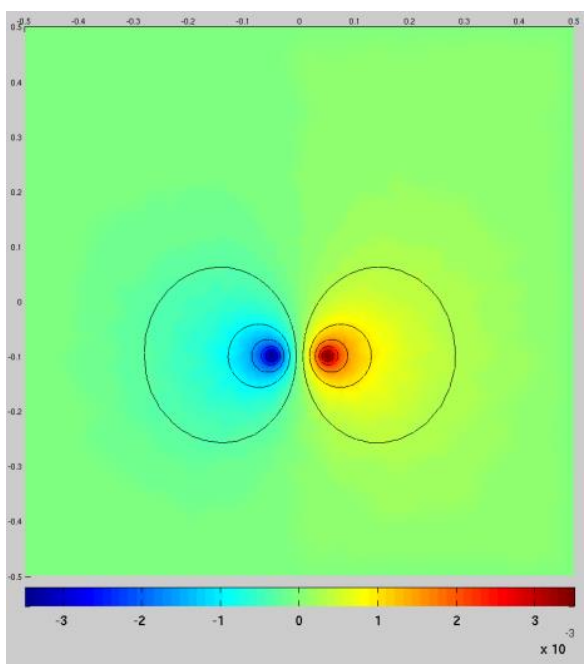

b)

Figure 4. Simulation results of excited coil

Comparing Fig. $4 a$ and $4 b$, it can be seen that the magnitude of the magnetic vector potential in the model are similar.

Fig. 5. shows the simulation result of the magnetic potential, where the model consists an excited one turned coil with ferrite core. Fig. 5a shows the simulation results in GMSH environment. Fig. 5b shows the simulation results in MATLAB environment. 


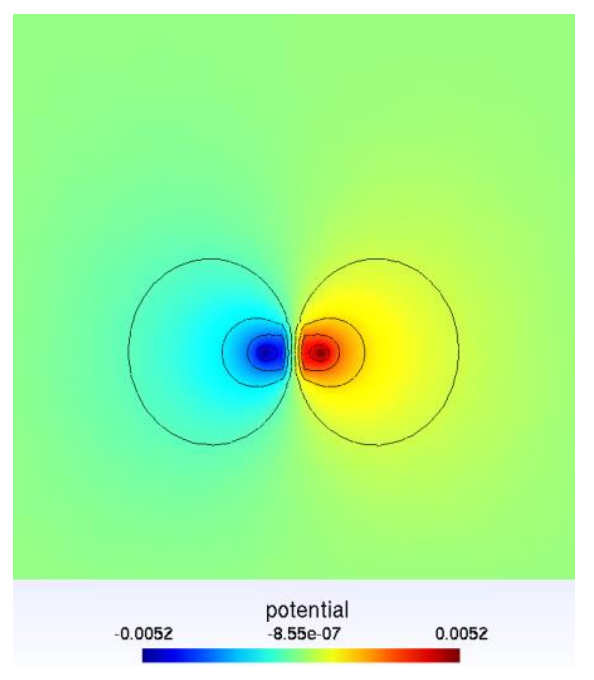

a)

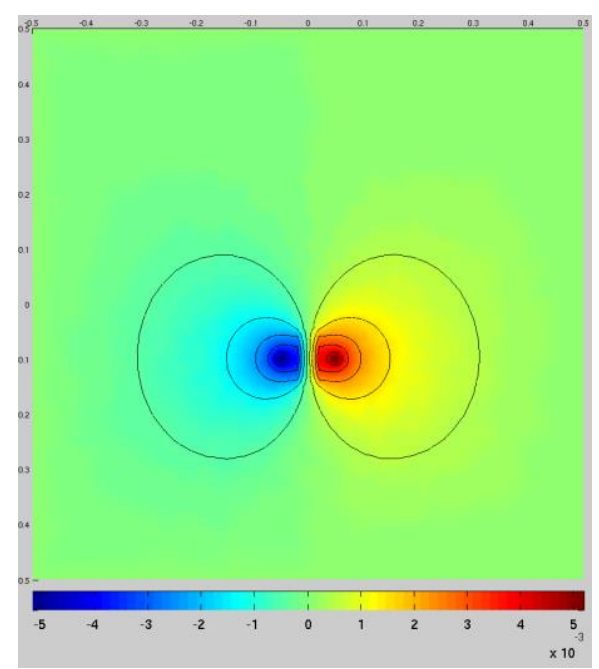

b)

Figure 5. Simulation results of excited coil with ferrite core

Comparing the Fig. 5a and 5b figures with each other, it can be seen that the magnitude of the magnetic vector potential in the model are similar.

Fig. 6. shows the simulation result of the magnetic potential, where the model consists a permanent magnet. Fig. 6a shows the simulation results in GMSH environment. Fig. 6b shows the simulation results in MATLAB environment.

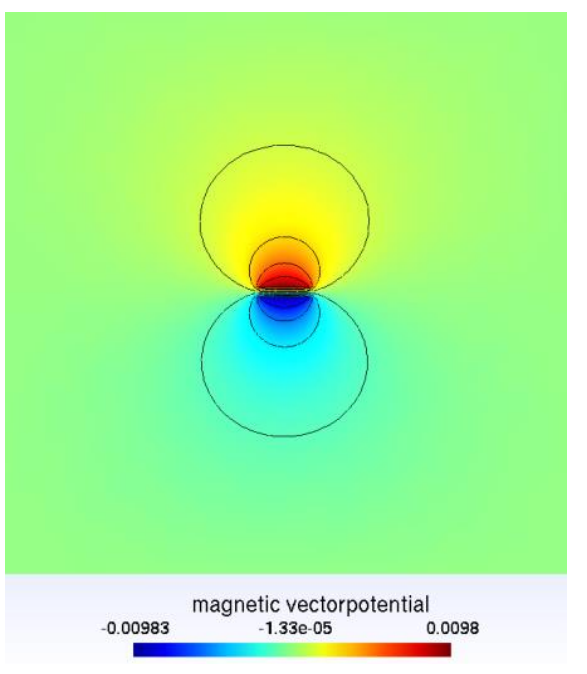

a)

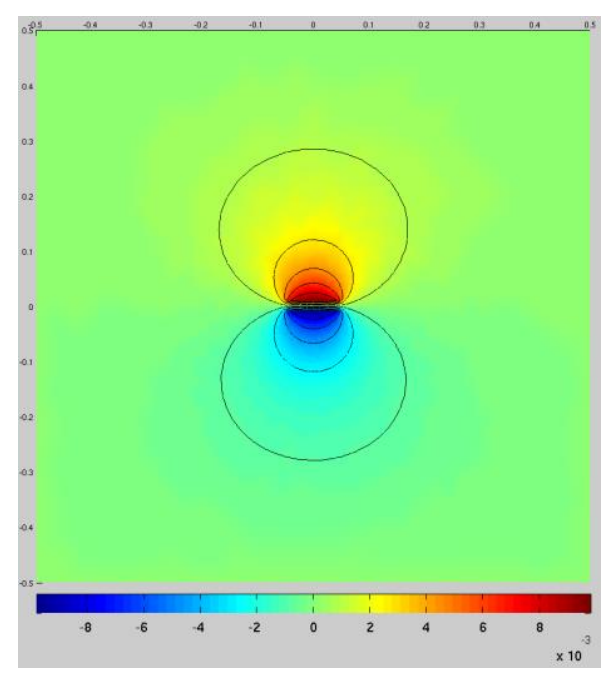

b)

Figure 6. Simulation results of magnet

Comparing the Fig. $6 \mathrm{a}$ and $6 \mathrm{~b}$ figures with each other, it can be seen that the magnitude of the magnetic vector potential in the model are similar. 
Fig. 7. shows the simulation result of the magnetic potential, where the model consists a permanent magnet with ferrite core. Fig. 7a shows the simulation results in GMSH environment. Fig. $7 \mathrm{~b}$ shows the simulation results in MATLAB environment.

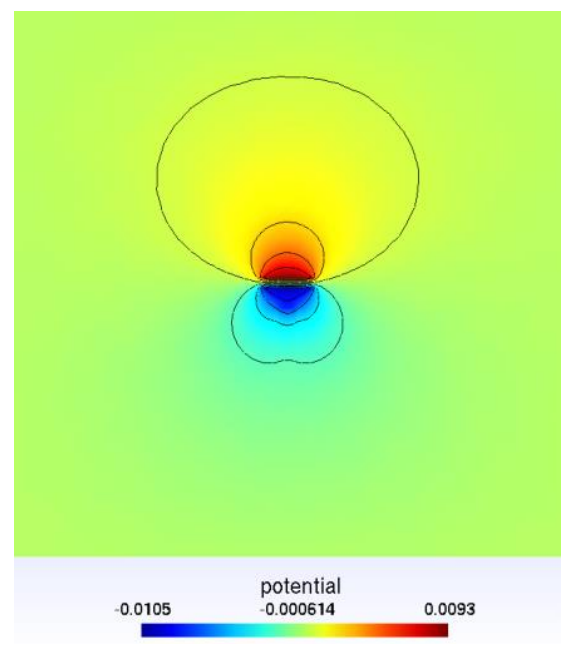

a)

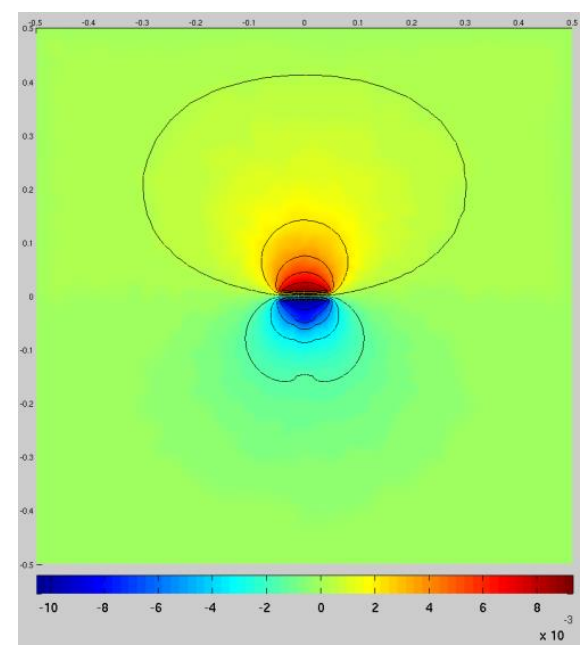

b)

Figure 7. Simulation results of magnet with ferrite core

Comparing the Fig. $7 \mathrm{a}$ and $7 \mathrm{~b}$, it can be seen that the magnitude of the magnetic vector potential in the model are similar.

Fig. 8. shows the simulation result of the magnetic potential, where the model consists of a permanent magnet with ferrite core and one turned excited coil. Fig. 8a shows the simulation results in GMSH environment. Fig. $8 \mathrm{~b}$ shows the simulation results in MATLAB environment.

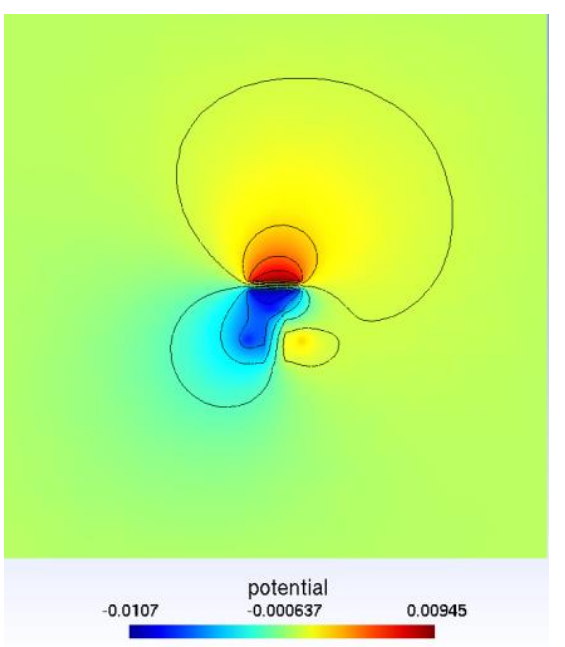

a)

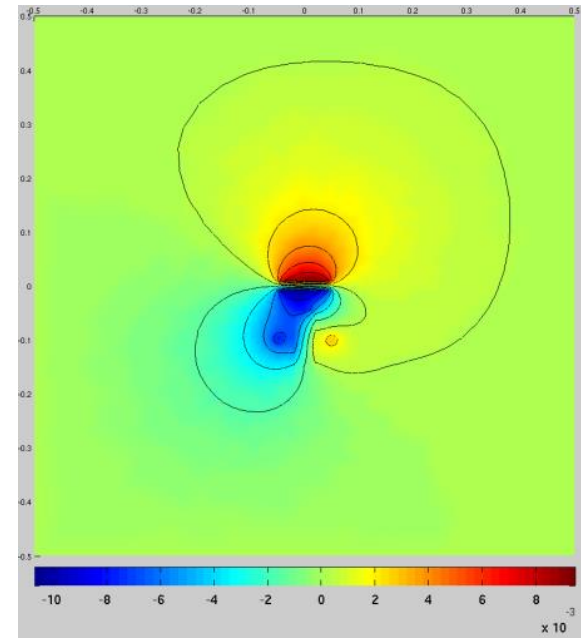

b)

Figure 8. Simulation results of magnet, ferrite core and excited coil 
Comparing the Fig. $8 \mathrm{a}$ and $8 \mathrm{~b}$, it can be seen that the magnitude of the magnetic vector potential in the model are similar.

\subsection{Differences of the simulation results}

Simulation results were also compared with each other focusing on the magnitude of the differences of models.

Fig. 9. shows the differences of the simulation results of the two different development environment in the case of the model of one turned excited coil.

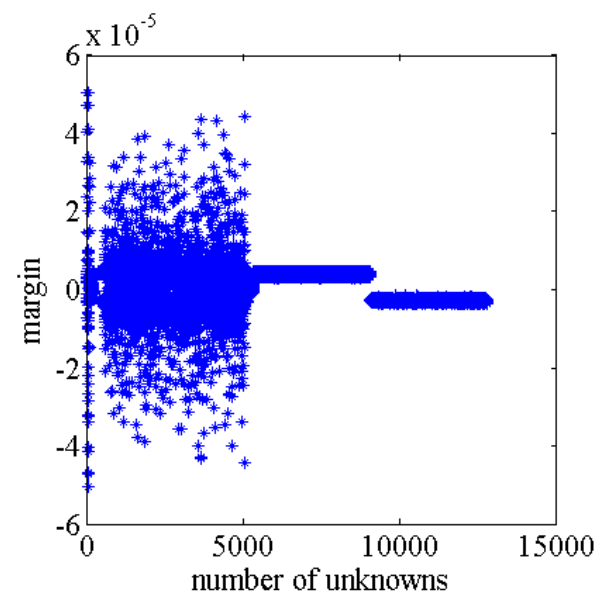

Figure 9. Margin between the simulation results, model: excited coil

The magnitude of the difference of the simulation results is about $10^{-5}$.

Fig. 10. shows the differences of the simulation results of the two different development environment in the case of the model of one turned excited coil with ferrite core.

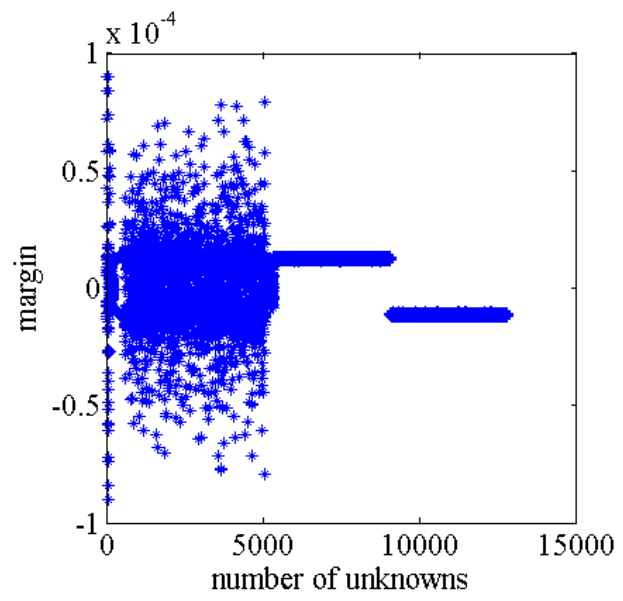

Figure 10. Margin between the simulation results, model: excited coil with ferrite core 
The magnitude of the difference of the simulation results is about $10^{-4}$.

Fig. 11. shows the differences of the simulation results of the two different development environment in the case of the model of permanent magnet.

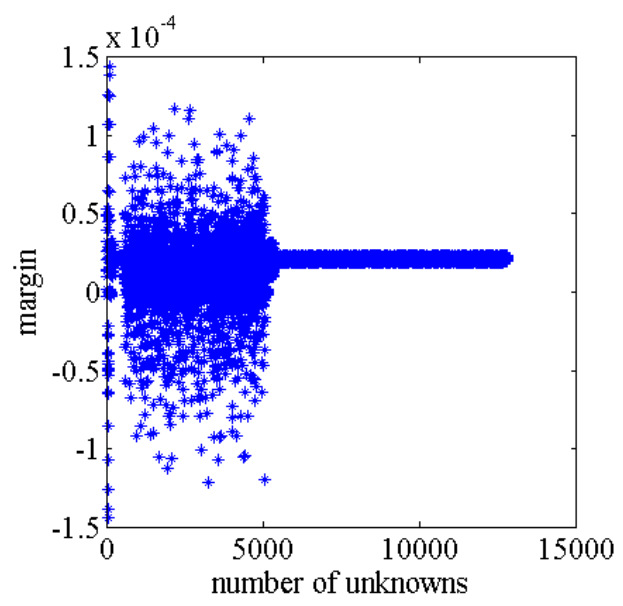

Figure 11. Margin between the simulation results, model: magnet

The magnitude of the difference of the simulation results is about $10^{-4}$.

Fig. 12. shows the differences of the simulation results of the two different development environment in the case of the model of permanent magnet with ferrite core.

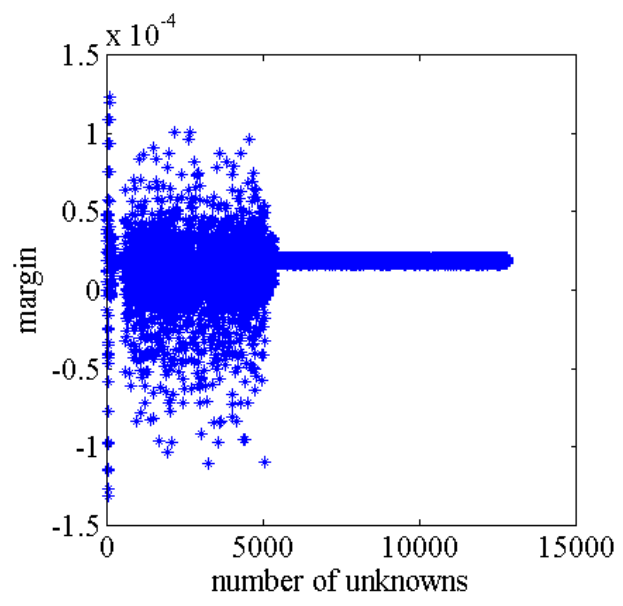

Figure 12. Margin between the simulation results, model: magnet with ferrite core

The magnitude of the difference of the simulation results is about $10^{-4}$.

Fig. 13. shows the differences of the simulation results of the two different development environment in the case of the model of permanent magnet with ferrite core and one turned excited coil. 


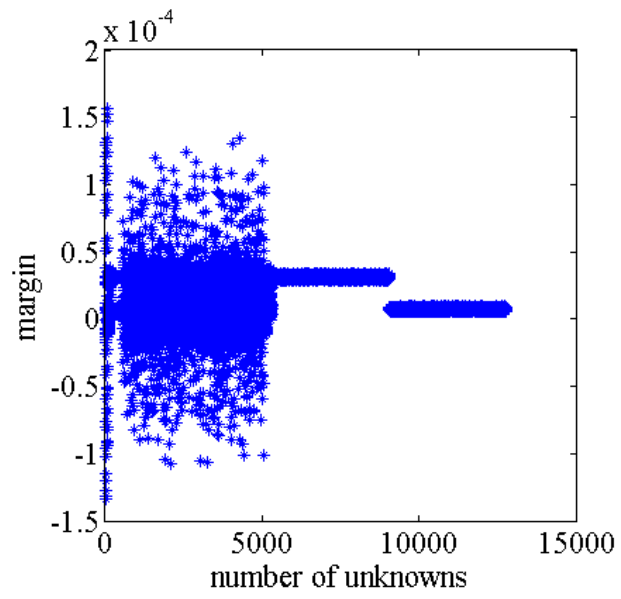

Figure 13. Margin between the simulation results, model: magnet, ferrite core and excited coil

The magnitude of the difference of the simulation results is about $10^{-4}$. It can be seen in every case that the differences of the simulation results are very small.

\section{Conclusion}

The new, finite element package based on $\mathrm{C}$ programming language is faster than the MATLAB solver, but the accuracy of the simulation results is adequate.

In the future work this $\mathrm{C}$ programming language based finite element package will be used for simulation and optimization of permanent magnet synchronous motors in a more fast and accurate way. This solution will have used in a research work where arrangements of the magnets and air gaps of the rotor of the BLDC motor to develop more energy efficient BLDC motors will have investigated.

\section{Acknowledgement}

This research was realized in the frames of TÁMOP 4.2.4. A/2-11-1-2012-0001 „National Excellence Program - Elaborating and operating an inland student and researcher personal support system convergence program" The project was subsidized by the European Union and co-financed by the European Social Fund.

\section{References}

[1] Geuzaine, C., Remacle, J.F.: Gmsh: a three-dimensional finite element mesh generator with built-in pre- and post-processing facilities, International Journal for Numerical Methods in Engineering, vol. 79, no. 11, pp. 1309-1331, 2009

DOI: $10.1002 / \mathrm{nme} .2579$

[2] Bianchi, N.: Electrical Machine Analysis Using Finite Elements, CRC Press, Boca Raton, 2005 
[3] Bastos, J.P.A., Sadowski, N.: Electromagnetic Modeling by Finite Element Methods, Marcel Dekker Inc., New York, Basel, 2003

[4] Gieras, J.F., Wang, R-J., Kamper, M.J.: Axial Flux Permanent Magnet Brushless Machines, (2nd Edition) Springer, 2008

[5] Kuczmann, M., Iványi, A.: The Finite Element Method in Magnetics, Akademic Press, Budapest, 2008

[6] M. A. Plonus: Applied Electromagnetics, McGraw-Hill, New York, 1978

[7] Bíró, O., Richter, K.R.: CAD in Electromagnetism, Advances in Electronics and Electron Physics, vol. 82, no. 1, pp. 1-91, 1991

DOI: 10.1016/S0065-2539(08)60911-7

[8] Kostaridis, A., Soras, C., Makios, V.: Magnetostatic Analysis of a Brushless DC motor Using a Two-Dimensional Partial Differential Equation Solver, Computer Applications in Engineering Education, vol. 9, no. 2, pp. 93-100, 2001 DOI: $10.1002 /$ cae. 1010

[9] Kumaravelu, U.D., Yakub, S.M.: Simulation of outer rotor permanent magnet brushless DC motor using finite element method for torque improvement, Journal Modelling and Simulation in Engineering, vol. 2012, article ID. 17, 6 pages, 2012 DOI: $10.1155 / 2012 / 961212$

[10] Caihong, Z., Hongtao, Z.: Magnet Field Finite Element Analysis of Permanent Magnet Brushless DC Motors based on ANSOFT, Advanced Materials Research, vol. 904, pp. 504-507, 2014

DOI: $10.4028 /$ www.scientific.net/AMR.904.504 